\title{
Haptic Feedback to Detect Obstacles in Multiple Regions for Visually Impaired and Blind People
}

\author{
Aaron Raymond See, ${ }^{1 *}$ Leonheart Van M. Costillas, ${ }^{1}$ \\ Welsey Daniel C. Advincula, ${ }^{1}$ and Nilo T. Bugtai ${ }^{2,3}$ \\ ${ }^{1}$ Department of Electrical Engineering, Southern Taiwan University of Science and Technology, \\ Tainan 71005, Taiwan \\ ${ }^{2}$ Manufacturing Engineering and Management Department, De La Salle University, \\ Manila, 1004 Metro Manila, Philippines \\ ${ }^{3}$ Institute of Biomedical Engineering and Health Technologies (IBEHT), De La Salle University, \\ Manila, 1004 Metro Manila, Philippines
}

(Received December 14, 2020; accepted January 27, 2021; online published March 31, 2021)

Keywords: visually impaired and blind people (VIBs), haptic interface, robot operating system (ROS), localized obstacle detection

Focus on the development of assistive devices for visually impaired and blind people (VIBs) to provide assistance in their safety and mobility has increased, but making such devices portable is still a challenge. We propose a system for localized obstacle avoidance with a haptic-based interface for VIBs implemented using a robotic operating system (ROS) to improve the obstacle detection of existing assistive devices. With a depth camera sensor, an obstacle localization algorithm was developed utilizing the ROS framework to identify key regions to detect head-level, left/right torso-level, and left/right ground-level obstacles. The proposed wearable device provides a discernible array of haptic feedback to convey the perceived locations of obstacles. The system was tested by blindfolded volunteers to determine the accuracy in determining object locations in various environments. Experimental results showed the consistency of the system across different setups. The obstacle detection algorithm was optimized and evaluated to discriminate noises and concurrently detect smaller obstacles, thus making detection more robust. Subsequently, the Eulerian video magnification method was used to determine the level of vibration isolation for a prototype.

\section{Introduction}

The increasing number of visually impaired and blind people (VIBs) has been highlighted by international organizations including the World Health Organization (WHO). ${ }^{(1,2)}$ It was estimated that there were more than 200 million people with various levels of visual impairment in 2020, and it was projected that by 2050 , the number of people with moderate to severe visual impairment will reach 550 million people. ${ }^{(3)}$ Those affected by visual impairment experience a drop in the quality of life due to limitations in activities they can do and the locations they are able to go to. ${ }^{(4)}$ Hence, assistive technologies have been developed by researchers and industry

*Corresponding author: e-mail: aaronsee@stust.edu.tw https://doi.org/10.18494/SAM.2021.3221 
to alleviate the struggles of VIBs. Currently, there are numerous solutions that try to tackle this problem, from traditional white canes used like an extended $\operatorname{arm}^{(5)}$ to wearable ultrasonic sensors that detect obstacle proximity ${ }^{(6)}$ and implants that stimulate the visual cortex to invoke "sight" in VIBs. ${ }^{(7)}$

However, most recently proposed assistive devices for the mobility of VIBs require multiple sensors, and their accuracy is greatly influenced by external conditions, the limited interface with the user, the inability to detect obstacles, especially at head level, and insufficient portability. ${ }^{(8-11)}$ In this paper, we used the robotic operating system (ROS) framework to develop a wearable assistive device for VIBs, which provides localized obstacle detection with a haptic-based interface to address the major gaps existing in assistive devices for the mobility of VIBs.

\section{Materials and Methods}

We implemented the obstacle detection and localization system to enable safe mobility for VIBs through the early detection of obstacles in their paths. A traditional device used by VIBs in their travels is the white cane, ${ }^{(12-14)}$ which can only detect obstacles on the ground and cannot detect obstacles such as hanging branches and other overhead obstacles. Although a sensor can be attached to a white cane to detect head-level obstructions, only binary data, i.e., an obstacle existing/not existing around the sensor, can be obtained without any location data. ${ }^{(15)}$ Hence, to overcome the limitations of existing devices, we implemented a region-based single sensor for obstacle detection, which provides more information to the user. A haptic feedback mechanism was developed for the sensor that provided one motor for each of the different regions of interest around the user.

\subsection{Proposed system architecture}

The ROS framework was used to develop the proposed system architecture. The ROS framework is a common environment in the field of robotics that is used for the development of scalable applications. It enables different programs to run simultaneously even when using different programming languages. It is also convenient for integrating these programs into a single architecture because it uses a message-passing type of communication, wherein a node publishes a language-neutral variable on a topic for use in any other node within the architecture. This message-passing type of communication allows the ROS framework to be a multilanguage architecture. ${ }^{(16,17)}$ The proposed architecture using the ROS framework was run on a Raspberry Pi 4 (RPi4) embedded system. The researchers made use of Ubiquity Robot OS, which runs on Ubuntu 16.04-based images and has an ROS pre-installed. Most of the nodes were developed using Python language and made use of both the ROS and Python 2.7 dependencies. Two ROS nodes were utilized for the system to implement the system workflow, namely, the depth image acquisition node and the obstacle localization node. 


\subsection{Depth image acquisition}

The system made use of an Intel RealSense D435i depth camera (Intel Corporation, California, U.S.A.) for its visual inputs. This camera enables the RGB and depth streams to be used in the different modules of the device. The depth information was obtained by using a dot projector and two infrared cameras at different positions, and the concept of triangulation was applied to calculate the depth. ${ }^{(18)}$ The value obtained by the depth sensor is the depth value, not the range. Hence, the depth pixel value is a measurement from the parallel plane of the viewer and not the absolute range. For the proposed system, we made use of the librealsense package developed by the ROS organization for rapid integration of the depth camera with the ROS environment.

\subsection{Obstacle localization}

The depth image captured by the sensor is pre-processed by applying a median filter to eliminate salt-and-pepper noise in the raw depth image stream. Then it is divided into five regions, namely, the left ground, right ground, left torso, right torso, and head levels, to cover the regions in which the user may encounter obstacles. The ground levels are covered by the white cane, while the upper levels correspond to regions where there may be suspended obstacles to the side of the user. ${ }^{(15)}$ The left and right regions for the torso and ground levels allow the user to react to obstacles on one side by moving in the opposite direction. The region setup within the depth frame is shown in Fig. 1. The obstacle detection algorithm detects not only the presence of obstacles in each region but also the distance between the user and obstacles. This is done by extracting the fifth percentile of the depth values for each pixel in a region.

\subsection{Haptic interface}

The implementation of the haptic interface is portrayed in Fig. 2, wherein each region is represented by a specific haptic motor embedded on a haptic lace. Martinez et al. found that

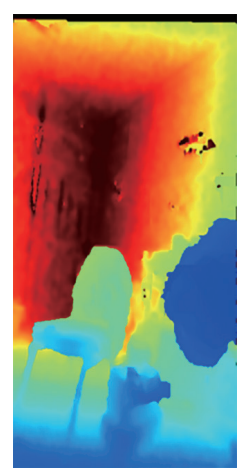

(a)

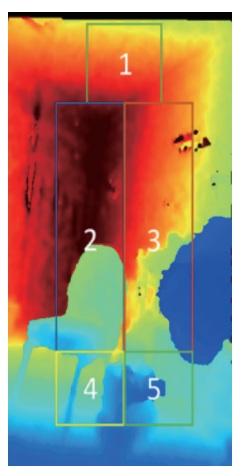

(b)

Fig. 1. (Color online) Obstacle detection designed to provide the user with (a) depth image perceived by Intel RealSense and (b) region assignment for obstacle detection along the user's path. 


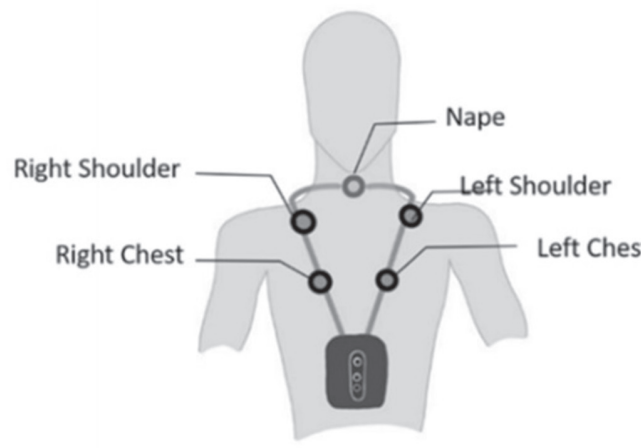

(a)

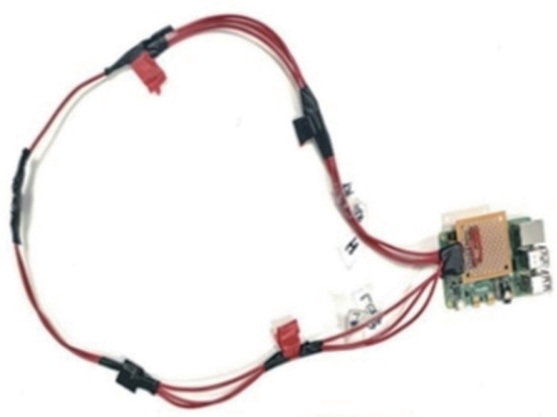

(b)

Fig. 2. (Color online) Haptic interface of the proposed system. (a) Placement of vibration motors on the user's body and (b) developed haptic hardware.

a haptic interface for mobility information is more efficient than using an auditory system, since the former can also be used as other interfaces such as for communications, textual notifications, etc. ${ }^{(19)}$ The GPIO pins of the RPi4 were used to trigger the controls for the haptic motors based on the output of the localization algorithm using pulse-width modulation (PWM) signals. A stronger vibration is felt whenever an obstacle is closer, and this is achieved by changing the operating voltage of each motor through the PWM signal.

\section{Results and Discussion}

\subsection{System component evaluation}

The accuracy of the Intel RealSense D435i depth readings was tested for different lighting conditions and distances to determine their reliability and whether a recalibration was required. The developed obstacle localization algorithm was evaluated on many distinct types of obstacles. Figure 3 shows a sample test input and the result of the algorithm, wherein Fig. 3(a) is a pseudo-colored plot of a depth frame in a region with a $1 \mathrm{~m}$ obstacle present in the frame and Fig. 3(b) shows a histogram of the input depth image, where the red line indicates the result of the algorithm. Subsequently, the results showed accurate distance measurement of the obstacle present for each region. For each scenario, 30 depth readings were accumulated per combination of distance and identified lighting condition, with the average shown in Table 1. Data on the different scenarios (distances and lighting conditions) showed results very close to the ground truth, demonstrating that the sensor is sufficiently accurate to be integrated with the proposed system without any recalibration.

A prototype of the developed system was developed, as shown in Fig. 4(a), with the location of the motor marked with a cross, and it was evaluated to verify the vibration isolation of each motor from the other parts of the prototype. The Eulerian video magnification technique developed by $\mathrm{Wu}$ et al. was utilized to visually detect the location and spread of vibration of each haptic motor in the prototype. The technique basically enhances the small vibrations in a 


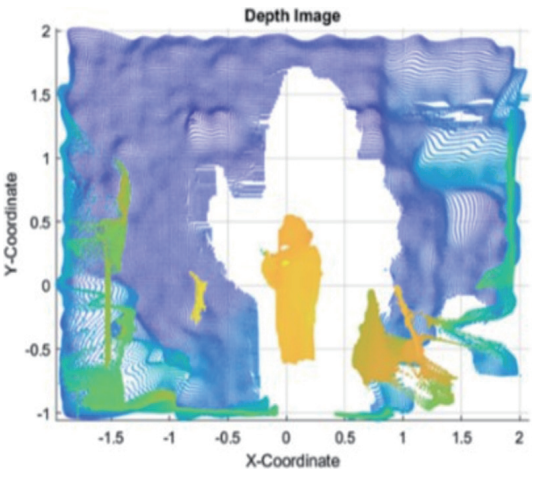

(a)

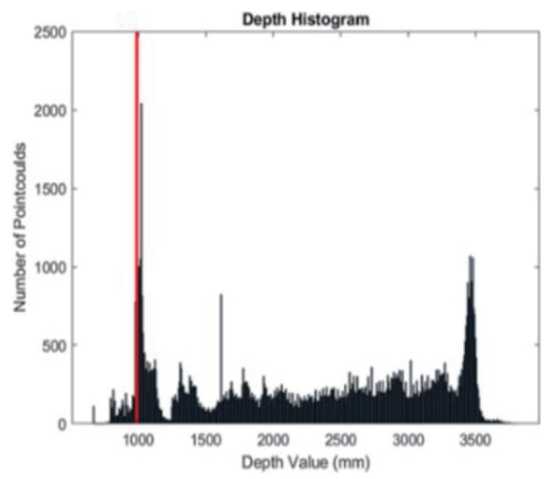

(b)

Fig. 3. (Color online) Obstacle localization results. (a) Depth frame input with $1 \mathrm{~m}$ obstacle and (b) histogram of the depth image, where the red line shows the distance of the detected obstacle.

Table 1

Average readings of depth sensor for different lighting conditions and distances.

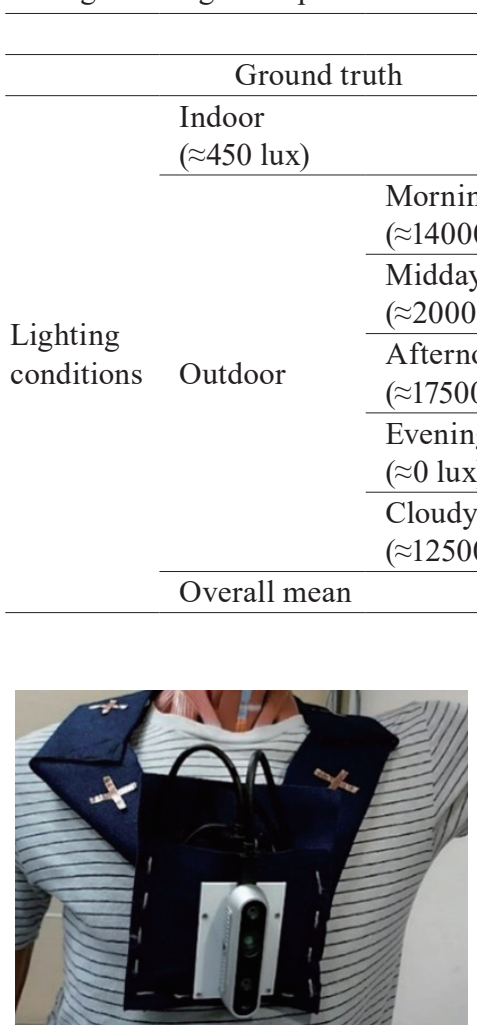

(a)

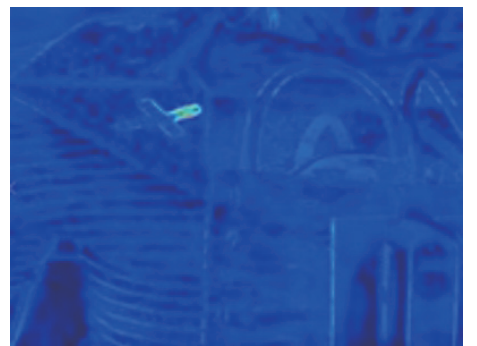

(b)

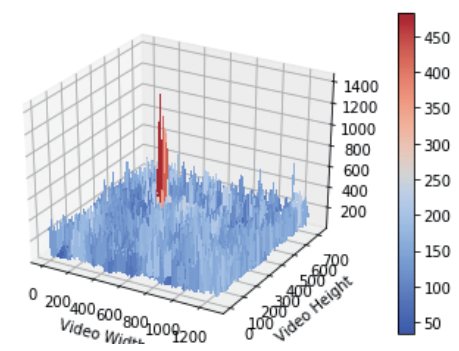

(c)

Fig. 4. (Color online) Vibration isolation test of the prototype using Eulerian video magnification. (a) System prototype, (b) pseudo-colored image of the vibration intensity in the setup, and (c) 3D graph of the accumulated per pixel intensity differences of the magnified and original videos.

video stream, which are originally indistinguishable by the naked eye, through the application of spatial decomposition, temporal filtering, and amplification of the frames in the video stream. ${ }^{(20)}$ A $20 \mathrm{~s}$ video of the prototype with the right ground motor turned on was recorded at $240 \mathrm{fps}$ 
to serve as input for the Eulerian video magnification method with the magnification set to 50. A per-frame comparison of the resulting magnified and original videos was done through calculation of the per-pixel grey level value differences of each frame. Figure 4(b) shows a pseudo-colored representation of the accumulated differences of the magnified and original videos and Fig. 4(c) shows a graph of the magnitude of the accumulated differences. According to these results, the prototype was clearly able to isolate the motor vibration relatively near to the location of the motor. This will allow the effective transfer of information about the obstacle detected by the system to the VIB user.

\subsection{Preliminary user testing}

An initial user evaluation of the system was conducted with 10 volunteers, five males and five females, with ages of 19-28. They were all normally sighted individuals who were blindfolded to conduct the test. It is important to note that we conducted minimal training of the test participants on the basic user instructions of the prototype prior to the evaluation. The evaluation has two main parts: identification of every pattern and combination of the haptic motors, and navigation in an obstacle course with different types of obstacles as shown in Fig. 5(a). The haptic implementation patterns and combinations are detailed in Table 2 and

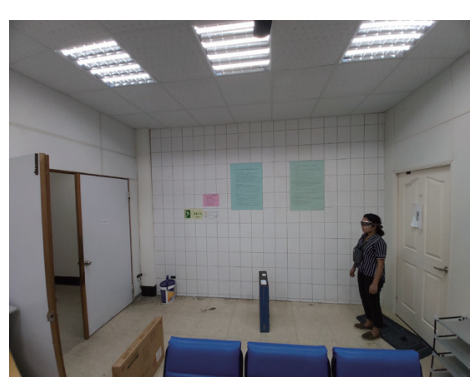

(a)

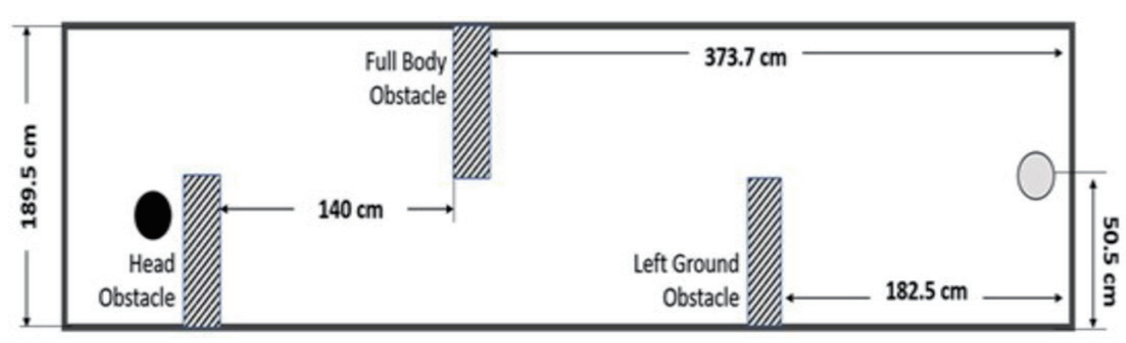

(b)

Fig. 5. (Color online) Experimental setup. (a) Actual experimental setup and (b) map of obstacle course.

Table 2

Interpretation of vibration patterns.

\begin{tabular}{lccc}
\hline Vibration pattern no. & Active vibration motors & Region assignment & Interpretation of obstacle detected \\
\hline 1 & Right chest & 4 & Right ground \\
2 & Right shoulder & 2 & Right torso \\
3 & Nape & 1 & Head level \\
4 & Left shoulder & 3 & Left torso \\
5 & Left ground & 5 & Left ground \\
6 & All & $1,2,3,4,5$ & Full body obstruction \\
7 & Right shoulder and right chest & 2,4 & Full right body obstruction \\
8 & Left shoulder and left chest & 3,5 & Full left body obstruction \\
9 & Right chest and left chest & 4,5 & Full ground obstruction \\
\hline
\end{tabular}


the researchers performed a 30-item test on the blindfolded volunteers on vibration pattern comprehension. For the second experiment, as seen in Fig. 5(b), the black ellipse is the starting point and the pale ellipse is the end point. Although there were several obstacles in the path, it was expected that the participant would be able to navigate around the obstacles and reach the intended destination. The participant moved forward by following the sound of the beacon at the finish point. For this experiment, the participant wore the prototype of the haptic lace and attempted to follow navigational instructions communicated through vibrations, moving away from obstacles perceived through vibration patterns by stepping to the left or right to navigate around them or through touching an obstacle in the head region to safely go under it.

Table 3 shows the results of the recorded 30-item test on vibration pattern comprehension. It shows that the users were able to perceive the correct location of the obstacle with an average accuracy of $85 \%$ and a reaction time of $2.28 \mathrm{~s}$. Vibration 3, which activates the vibration motor in the user's nape, showed the highest accuracy and had the quickest response time. This may be because of the sensitivity of the nape area, which can easily be distinguished from the other locations of the body where the vibration motors are placed. Vibration 9, which activates vibration motors located on the right and left chest areas, had the slowest reaction time, and this may be due to the participants being extra careful in determining whether the vibration was from just one of the chest areas or both. On average, the vibration patterns involving the activation of multiple vibration motors (Vibrations 6, 7, 8, and 9) were less accurate than those involving the activation of a single vibration motor. This is because all the participants had minimal training on using the prototype prior to the experimentation; thus, most of the participants tended to focus on the region where they felt the strongest vibration and could not always perceive other vibrating motors. However, as the participants got used to the device, it was observed that their comprehension of each vibration pattern combination improved, which is supported by the results in the subsequent experiment involving obstacle course navigation. In the obstacle course navigation, the system was able to accurately detect the obstacles in the course and effectively communicate them to the test participants through the haptic lace. Given the overall satisfactory response time of all participants in the first test, they found it easy to react to any obstacle by immediately stopping, followed by comprehension of the vibration patterns and necessary adjustments to navigate around all types of obstacles.

Table 3

Average correct vibration recognition and average response time to vibration patterns.

\begin{tabular}{lcc}
\hline Vibration pattern & Average correct answers (\%) & Average reaction time (s) \\
\hline Vibration 1 & 88.9 & 2.51 \\
Vibration 2 & 80.0 & 2.38 \\
Vibration 3 & 100.0 & 1.87 \\
Vibration 4 & 86.7 & 2.49 \\
Vibration 5 & 93.0 & 2.49 \\
Vibration 6 & 88.9 & 1.93 \\
Vibration 7 & 59.3 & 2.25 \\
Vibration 8 & 73.3 & 2.24 \\
Vibration 9 & 81.5 & 2.79 \\
\hline Overall & 85.0 & 2.28 \\
\hline
\end{tabular}




\section{Conclusions}

Existing assistive devices to improve the safety and mobility of VIBs still have limitations. The requirement of multiple sensors, the degree of accuracy, the limited user interface, the detection of types of obstacles, especially head-level obstacles, and portability are several aspects in which related assistive technologies are still unsatisfactory. Thus, there is still a need for improvements and innovations in these areas through the integration of state-of-theart technologies. We developed a wearable assistive device that addresses the above-mentioned limitations and presents a viable solution through the application of modern technologies. This was achieved through implementing an ROS-based system architecture on a wearable assistive device that can localize different types of obstacles through region-based obstacle detection with an intuitive haptic interface. The reliability of the proposed system was evaluated by conducting multiple system component assessments. The depth camera sensor showed high distancereading accuracy, not only for different distances but also for different lighting conditions. The localization algorithm was also proven to be robust through testing with different types of obstacles. The haptic interface of the prototype was also assessed to determine the degree of motor vibration isolation using the Eulerian video magnification method, with results showing very well isolated vibration. After obtaining these promising results for the system, initial user testing was conducted on 10 blindfolded participants. An average accuracy of $85 \%$ was obtained for the perception of different vibration patterns and combinations with a mean response time of $2.28 \mathrm{~s}$, and all participants were easily able to complete an obstacle course navigation task relatively quickly. The results indicate not only the reliability of the system but also its comprehensiveness, making it easy to use. Further evaluation was planned with actual VIB users to further improve the system, which has been delayed owing to the current COVID-19 pandemic.

\section{Acknowledgments}

This research was funded by the Ministry of Science and Technology of Taiwan (grant numbers MOST 109-2222-E-218-001-MY2 and MOE 1300-108P097). We would like to thank Resources for the Blind in the Philippines and Tainan City You Ming Visually Impaired Association for their inputs, which gave us insights and inspiration for the project.

\section{References}

1 W. H. Organization: Blindness and Vision Impairment, https://www.who.int/news-room/fact-sheets/detail/ blindness-and-visual-impairment (accessed September 2019).

2 R. R. Bourne, S. R. Flaxman, T. Braithwaite, M. V. Cicinelli, A. Das, J. B. Jonas, J. Keeffe, J. H. Kempen, J. Leasher, and H. Limburg: Lancet Global Health 5 (2017) e888. https://doi.org/10.1016/S2214-109X(17)30293-0

3 T. Mazumdar: Global Blindness Set to 'Triple by 2050', https://www.bbc.com/news/health-40806253 (accessed September 2019).

4 R. Bourne, S. Resnikoff, and P. Ackland: Gbvi - Changes in Numbers \& Prevalence, http://atlas.iapb.org/ global-burden-vision-impairment/gbvi-changes-numbers-prevalence/ (accessed September 30, 2019).

5 P. Kelley, R. Davidson, M. Sanspree: J. Visual Impairment Blindness 87 (1993) 397. https://doi. org/10.1177/0145482X9308701004 
6 J. A. Hossack, J. S. Ha, and T. S. Sumanaweera: Medical Imaging 2001: Ultrasonic Imaging Signal Process. 4325 (2001) 102. https://doi.org/10.1117/12.428230

7 A. Kusnyerik, M. Resch, H. J. Kiss, and J. Nemeth: Vision Restoration with Implants: In Mobility of Visually Impaired People: Fundamentals and ICT Assistive Technologies (Springer International Publishing, Cham, 2018) p. 617. http://doi.org/10.1007/978-3-319-54446-5_20.

8 R. K. Katzschmann, B. Araki, and D. Rus: IEEE Trans. Neural Syst. Rehabil. Eng. 26 (2018) 583. https://doi. org/10.1109/TNSRE.2018.2800665.

9 S. Kayukawa, K. Higuchi, J. Guerreiro, S. Morishima, Y. Sato, K. Kitani, and C. Asakawa: Proc. 2019 CHI Conf. Human Factors in Computing Systems (2019) 1. https://doi.org/10.1145/3290605.3300282.

10 B. Li, J. P. Munoz, X. Rong, J. Xiao, Y. Tian, and A. Arditi: European Conf. Computer Vision (2016) 448. https://doi.org/10.1109/TMC.2018.2842751.

11 H.-C. Wang, R. K. Katzschmann, S. Teng, B. Araki, L. Giarré, and D. Rus: 2017 IEEE Int. Conf. Robotics and Automation (ICRA) (2017) 6533. https://doi.org/10.1109/ICRA.2017.7989772.

12 M. D. Messaoudi, B.-A. J. Menelas, and H. Mcheick: Technologies 8 (2020) 37. https://doi.org/10.3390/ technologies 8030037 .

13 Q. Dang, Y. Chee, D. Pham, and Y. Suh: Sensors 16 (2016) 95. https://doi.org/10.3390/s16010095.

14 T. Pallejà, M. Tresanchez, M. Teixidó, and J. Palacin: Sensors 10 (2010) 11322. https://doi.org/10.3390/ s101211322.

15 J. Foucault, S. Lesecq, G. Dudnik, M. Correvon, R. O’Keeffe, V. Di Palma, M. Passoni, F. Quaglia, L. Ouvry, and S. Buckley: Sensors 19 (2019) 4350. https://doi.org/10.3390/s19194350.

16 M. Quigley, K. Conley, B. Gerkey, J. Faust, T. Foote, J. Leibs, R. Wheeler, and A. Y. Ng: ICRA Workshop on Open Source Software 3 (2009) 5.

17 V. A. Hax, N. L. Duarte Filho, S. S. da Costa Botelho, and O. M. Mendizabal: J. Appl. Comput. Res. 2 (2013) 91. https://doi.org/10.4013/jacr.2012.22.05

18 A. Grunnet-Jepsen, J. N. Sweetser, P. Winer, A. Takagi, and J. Woodfill: Projectors for Intel ${ }^{\circledR}$ RealSense ${ }^{\mathrm{TM}}$ Depth Cameras D4xx; Intel Support, Intel Corporation: Santa Clara, CA, USA (2018).

19 M. Martinez, A. Constantinescu, B. Schauerte, D. Koester, and R. Stiefelhagen: Int. Conf. Computers for Handicapped Persons (2014) 128.

20 H.-Y. Wu, M. Rubinstein, E. Shih, J. Guttag, F. Durand, and W. Freeman: ACM Trans. Graphics 31 (2012) 1. https://doi.org/10.1145/2185520.2185561.

\section{About the Authors}

Aaron Raymond See is an assistant professor in the Department of Electrical Engineering, Southern Taiwan University of Science and Technology. He concurrently holds the position of Director of Student Affairs in the Office of International Affairs. He was born in Manila, Philippines, and received his B.S. degree in electronics and communications engineering from De La Salle University, Manila, in 2006. He obtained his master's and Ph.D. degrees in electrical engineering with a major in biomedical engineering from Southern Taiwan University of Science and Technology in 2010 and 2014, respectively. He runs courses on assistive technology device design and engineering education curriculum transformation. His research interests are in assistive device design and development, biomedical image and signal processing, and engineering education.

Leonheart Van M. Costillas received his B.S. degree in electronics engineering with specialization in instrumentation and control from Cebu Institute Technology - University, Cebu City, Philippines, in 2017. He is currently pursuing his master's degree in electrical engineering at Southern Taiwan University of Science and Technology, Taiwan. He primarily developed his curiosity towards physics and engineering during his secondary education at Philippine Science High School - Eastern Visayas Campus, which later led to his current research interests in telecommunication systems design, instrumentation and control, embedded control systems, biomedical engineering, mobile application development, and robotics. 
Welsey Daniel C. Advincula received his B.S. degree in manufacturing engineering and management with a specialization in biomedical engineering from De La Salle University, Manila, Philippines, in 2019, and is currently pursuing his M.S. degree in electrical engineering at Southern Taiwan University of Science and Technology, Tainan, Taiwan. His current research interests include biomedical engineering, haptic control, and computer vision.

Nilo T. Bugtai holds a B.S. degree in mechanical engineering from the University of San Jose - Recoletos, Cebu City, Philippines, an M.S. degree in manufacturing engineering as a DOSTPCIERD Scholar from De La Salle University (DLSU), Manila, Philippines, and a Ph.D. degree in manufacturing as a DOST-ESEP Scholar from Loughborough University, U.K. (2003). He is currently a technical expert at De La Salle University Innovation and Technology Office (DITO). He has completed several special studies and courses since 2005 on IP auditing, IP protection, IP management, and IP training held locally and abroad. He has presented several technical and research papers at local and international conferences and published them in local and international journals. He has been the recipient of a multimillion-peso Grant-in-Aid since 2016 from the Department of Science and Technology, Philippine Council for Health Research and Development (DOST-PCHRD), to increase innovation in research in the Philippines on biomedical devices, especially in the fields of robotics, rehabilitation devices, and assistive technologies. He also received a Grant-in-Aid European Union (EU) Asia Link Program to develop a biomedical engineering program in the Philippines. He was awarded a prestigious Leaders in Innovation Fellowship (LIF) in 2017 and a grant from the LIF Advance Programme in 2020 by the Newton Fund of the British Council and the Royal Academy of Engineering in London, U.K. Currently, he is the director of the Institute of Biomedical Engineering \& Health Technologies and a full professor of manufacturing engineering \& management (MEM) of De La Salle University. 